\title{
Report from the Evolving Open Solutions (2) Workgroup
}

\author{
Marilyn Billings, Brett Bobley, Aaron McCollough, Alison Mudditt, Kamran Naim, Frances Pinter, \\ Richard Price, Tony Roche, Lisa Spiro, Micah V andegrift
}

\section{OSI2016 Workgroup Question}

Are the scholarly publishing tools we're using today still the right ones? Is the monograph still the best format in the humanities? Is the journal article still best in STM? These products can be difficult to produce and edit, nearly impenetrable to read, and-as in the case of clinical research information - they aren't necessarily the best-suited formats for capturing every piece of necessary information (like protocols and datasets in medical research) and showing how this information is all connected to other scholarship. What other formats and options are being considered or used? What are the prospects of change? How about the stakeholder universe itself? How are roles, responsibilities and expectations changing (and where might they end up)? Are we "settling" on half-measures or on the best possible solutions?

\section{Executive Summary}

The Evolving Open Solutions (2) workgroup (which we quickly renamed Revolution Solutions) structured our work at the inaugural Open Scholarship Initiative meeting in Fairfax, VA, April 19-22, 2016, around several focal points. ${ }^{1} \mathrm{We}$ agreed early that "evolution" presented too long a view and that what is necessary is revolution. The team imagined solutions that included, and often focused on, revolutions in monograph publishing, where the infrastructure for openness is lagging behind journal-focused disciplines. As a group, we identified the fact that changes in research culture (especially evaluation) must be researcher-driven and recognized by University administration, both of whom as stakeholders were largely absent from the meeting. Finally, we devoted time to imagining how funding models should be revolutionized to make room for experimentation and innovation in scholarly publishing.

Our group dynamic was spurred by the fact that we represented libraries, journal publishers, a research funder, university presses, a publishing start-up, and a research-sharing platform, with various levels of seniority within those organizations. Several members of the at-large workgroup joined us throughout the week, with invaluable contributions, particularly because of their insights as researchers and/or publishing consultants. Generally, our deliberations were focused and productive, although not without the expected measure of acknowledgement that our primary interests in this conversation might not always be in alignment.

The solutions we proposed grew from a compilation of the most significant challenges academic publishing must overcome in the next 3-5 years: 
- Bridge funding

- Are Article Processing Charges (APC's) the best or only way to move from subscription to open? Are there other solutions for disciplines and countries that are resource-scarce?

- Digital, open back-catalog

O How to get the back-catalog of publications online, freely available (e.g., an open access JSTOR)

- Researcher buy-in

o Revolution must begin with researcher buy-in of the benefits from and need for change.

- Researcher Information Management Systems (Converis, Symplectic Elements, PURE, VIVO)

- What role will these play in evaluation, leading to broader (open) conception of "impact"?

- 21st century publishing infrastructure

- What are the core infrastructural elements of a 21 st century publishing ecosystem (ORCID, unique IDs, data standards, etc.)?

- How do extramural research platforms integrate (e.g., Academia.edu feeding institutional repositories)?

- Understanding of the supply chain of research

O What are the costs vs. the price? Where do the costs occur and can they be alleviated? Does open access (OA) save on cost and reduce price?

- Separate spheres of "publishing"

○ Journals vs. monographs vs. all other research products

- What do we save from the past, and what do we need to let go of?

- How do we value, present and preserve new forms of research products (e.g., digital humanities projects)?

- Redefining the "community" of research consumers

O Who are the audiences? How should open scholarship be aimed or guided to these new consumers?

- System breakdown

$\circ$ Is dissolution the only way forward?

- Experimentation

o Partnerships with different stakeholders, exploring new models of sustainability

- Cultural challenges within academe

O What will drive institutional leaders, especially provosts, to acknowledge and push revolutionary change?

Our key takeaway at the close of day one was that significant culture change is necessary at all levels of the university (graduate students, researchers, department heads, deans, provosts, librarians) in order for publishing to evolve towards greater openness. This depth and breadth of cultural change will be enormously challenging, requiring strong coordination and great marketing. We identified four core components:

- Shifts in the incentive and reward structures to align with the goals of openness (especially in faculty evaluation)

- Shared infrastructure/ecosystems that are built, managed, and co-hosted by libraries, university presses, and publishers

- In-depth understanding of audiences and their needs/concerns

- New models of funding and spending 
The solutions proposed by our workgroup were compiled into three main categories: culture change, funding/sustainability, and expanded infrastructure.

\section{Themes}

\section{Culture Change}

Our workgroup recognized the powerful, positive benefits of openness in scholarly research but also acknowledged that these benefits were not necessarily accepted or understood by the major stakeholders at universities, particularly by university administrators or by many academics and researchers. We further discussed the tension between success as a researcher, the openness of scholarly research, and the need for reform in our academic evaluation and incentive systems. While the momentum around OA is certainly growing, awareness of the potential of $\mathrm{OA}$ to positively impact scholarship remains low among academics and administrators. And even when its benefit is recognized, OA rarely ranks as an important factor for scholars in deciding where to publish.

Furthermore, prevailing myths around open access (i.e. notions that it implies low quality, lack of peer review, etc.) inhibit changes in behavior among these stakeholders to advance openness; these misperceptions need to be dispelled through targeted outreach and education. We also recognized that behavioral change requires more than just outreach; a change in incentive structures will almost surely be required. The emergence of articlelevel metrics holds tremendous promise to provide a new set of measures that research evaluators can use to assess impact, and may encourage authors to more readily adopt OA publishing practices. There are already many success stories, for ex- ample, that can be shared about the ways in which OA models have extended the audience and impact of monographs well beyond the few hundred that most sell to wealthy Western libraries under the traditional, closed model.

The adoption of reformed practices for faculty performance evaluation and new incentive structures means that institutional-level cultural change will be necessary at all levels of academia: from university provosts, department heads, and deans to faculty and researchers. Of all these stakeholder groups, university administrators and provosts are unique in their potential to influence behavior and dynamics across the remaining groups, not least from their ability to effect policy changes and allocate financial resources. University administrators therefore have a critical role to play as champions of OA and leaders in driving cultural change around openness.

\section{Funding/Sustainability}

The widely documented decline of library budgets-and the increasing proportion of budget spent on subscription costswas identified as a significant obstacle for advancing OA. The dominant Gold model for OA is in many cases simply placing greater financial demands on libraries, which are now looked to for APC funding without any decrease in subscription spending; all while they are working to optimize the services they deliver to students and researchers while being confronted by diminishing resources. So although the APC model via both fullyopen and hybrid journals is increasingly accepted in fields that have substantial research grants, significant questions remain about its viability for other fields and types of research output (such as mono- 
graphs), not to mention for other parts of the globe, where an APC model addresses the issue of access but not that of participation.

While there are a number of alternate OA models emerging, much of the funding available to libraries is inflexible, with a bulk of resources locked up in "big deals" with major commercial publishers. Our workgroup recognized economic analyses, particularly the study by Max Planck in 2015, which demonstrated that there is more than enough money in the subscription market-some $\$ 10$ billion in journals alone-to underwrite open access. ${ }^{2}$ But moving from the current status quo to a fully "flipped" model requires a major shift in how institutions spend their limited-and largely fixed-funds on scholarly communication. On the one hand, this redirection from subscription spending (and especially "big deals") is a simple administrative adjustment, but as already acknowledged, scholarly communication is an entrenched evaluation system. Such 'administrative' changes quickly become mired in the wider cultural environment.

We further recognized the emergence of newer models that aim to leverage existing resources through the growing collaboration of publishers, libraries, and funders in supporting of open access publishing. These newer models extend from the sciences (e.g. SCOAP3) to the social sciences and humanities, as demonstrated by the examples of Knowledge Unlatched and the Open Library of the Humanities, among others. As promising as these new examples are, they present further challenges for libraries in making their "open access collection development" decisions. In the absence of other sources of funding, libraries are increasingly being asked to pitch in to support a plethora of new initiatives, raising concerns for us about the scalability of these resource reallocations. Available funding is already very limited, so how can we work together most effectively to consolidate available resources and advance innovative, effective and scalable approaches towards sustainable $\mathrm{OA}$ ?

\section{Infrastructure}

While publishers and other stakeholders have been highly effective in building shared infrastructure for digital and OA journals (Crossref, ORCID, CHORUS, etc.), open access monograph publishing remains a relatively new business. Multiple experiments-including the "unlatching" model coordinated by Knowledge Unlatched, the Luminos model from the University of California Press, and many smaller efforts from university presses and independent publishers-have begun to expose the unique infrastructural requirements for different disciplines and diverse forms of research output. Moreover, the development of that shared infrastructure is still at a very early stage.

Some of the critical gaps in the current infrastructure include the following:

- Discoverability: how to get records into both web-scale and local library discovery tools when OA content sits outside traditional workflows;

- Aggregations: models to include OA content in aggregated packages (EBSCO, MUSE, JSTOR and more) - again, to enhance discoverability;

- Long-term preservation, especially for output that includes media-rich content; 
- Publishing/hosting platforms that can handle OA content;

- Tools to aggregate usage data (critical to demonstrate the greater impact of OA models).

While there are a number of initiatives underway to support pieces of this infrastructure development (many of which are generously funded by the Andrew W. Mellon Foundation), we remain concerned about the lack of coordination and collaboration. The journal initiatives noted above were successful because the key stakeholders came together to identify gaps and needs, and then worked collaboratively to develop shared solutions. In contrast, current approaches to address the gaps we identify above pose the risk of patchwork results for scholarship, where cost-effective, scalable solutions are needed more than ever.

\section{Proposals}

\section{Cultural challenges and audience(s)}

With the recognition that dramatic change in the context of traditional scholarly publishing is a tremendously challenging venture complicated by a number of interwoven factors, our workgroup suggests that a targeted program to promote culture-change across institutional stakeholders is critical in order to advance Open Access.

\section{Recommendations}

- We recommend that OSI commission the development of a comprehensive set of resources and messaging efforts, targeted to specific audiences, to increase the profile of OA across stakeholder groups. These marketing resources should be both bottom-up (expanding on the good work already done by many librarians and other advocacy groups), as well as top-down, with targeted, research-supported, impact-driven storytelling that will increase engagement among senior administrators.

- We recommend the forming of "tiger teams" of committed and engaged OA champions, who can meet with key stakeholders on their turf to increase their knowledge of and engagement in OA. For example, these tiger teams could meet with faculty members, professors, and researchers at disciplinary conferences, or target meetings of university presidents and provosts, where stories of university level successes of OA could be celebrated.

\section{Funding}

As mentioned previously, library budgets are somewhat inflexible, with the bulk of financial resources locked into inefficient, commercial "big deals" and with few resources available to support Open Access initiatives. Although new OA models are emerging beyond hybrid and APC-based models, many of these are small pilots that lack scale and efficiency. As libraries are approached by multiple open access initiatives for funding, they often struggle to determine what initiatives to support and spread limited funding thinly.

We propose the collective establishment of an Open Access Venture Fund, financed by contributions from universities and libraries (among others), which would provide financial and managerial oversight and strategic expertise to new OA programs that propose innovative, viable ideas and promise the greatest potential for impact. The VC fund will be tasked 
with catalyzing new business models and technologies that deliver novel, high-risk innovations with the potential not only to disrupt the prevailing, entrenched models that stifle openness in the scholarly publishing industry, but will lead to sustainable, scalable open access publishing solutions.

Next, in order to liberate subscription dollars, and give libraries greater financial freedom to reprioritize their budgets in favor of OA, we propose a (long overdue) coordinated effort by libraries working with commercial publishers to phase out the inefficient "big deal" subscription model.

\section{Recommendations}

- We recommend that OSI appoint a Task Force to develop a strategy for the establishment of an $\mathrm{OA}$ venture fund, and deliver a report at OSI 2017. This Task Force will examine potential sources of venture funding (e.g., libraries, other institutional funds, research funders, commercial publishers, etc.). The Task Force will also be asked to identify specialized experts who have the skills to select, monitor, and support investments that reduce risk for investors into the $\mathrm{VC}$ funds.

- We recommend that the topic of liberating subscription budgets (and the dissolution of "big deal" models) be a future OSI Working Group, with representation from both libraries and publishers.

\section{Infrastructure}

Our workgroup identified several infrastructure limitations, which collectively limit the promulgation of open workflows and publication. In particular, these are:

- Lack of developed infrastructure beyond (STM) journals;

- Fragmentation and lack of interoperability of systems and processes.

\section{Recommendations}

- We recommend that an OSI Working Group identify and seek ways to close gaps within the OA infrastructure, beyond STM journals.

- We recommend a coordinated approach to leverage work that has already taken place or is ongoing. For example, OSI should consider working in partnership with OAPEN.

\section{Conclusion}

In order to determine support among OSI participants for our proposals, we ran a poll during the final session. We had 38 responses, with the most votes going to cultural change and redirecting subscription dollars. 


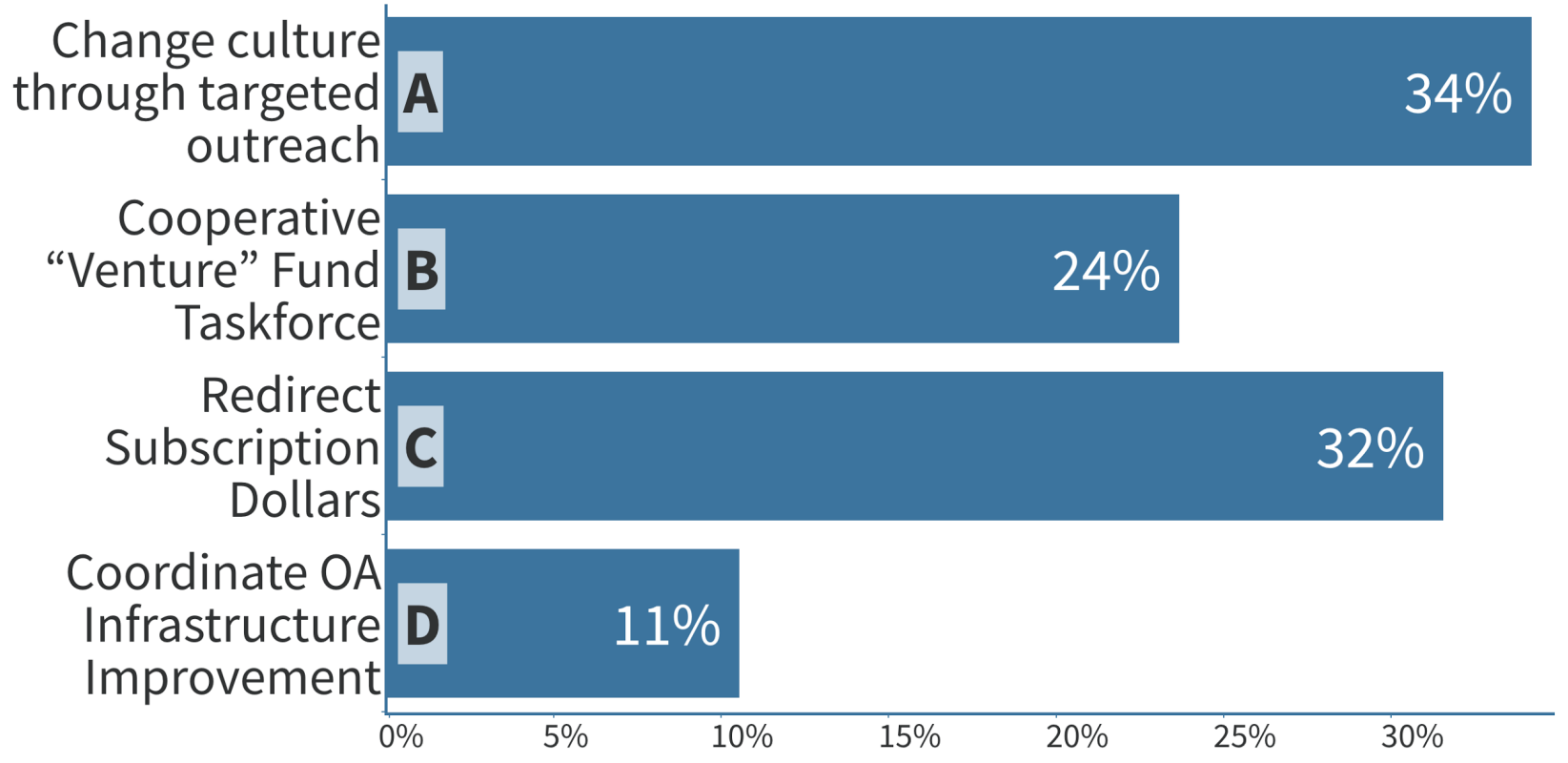

\section{OSI Evolving Open Solutions Working Group 2}

Marilyn Billings, Scholarly Communication \& Special Initiatives Librarian, University of Massachusetts,

Brett Bobley, CIO, National Endowment for the Humanities

Aaron McCollough, Head of Scholarly Communication and Publishing Unit, University of Illinois Library

Alison Mudditt, Director, University of California Press

Kamran Naim, Lead Researcher, Open Access Cooperative Publishing Study, Stanford University

Frances Pinter, Founder, Knowledge Unlatched, and CEO, Manchester University Press

Richard Price, Founder and CEO, Academia.edu

Tony Roche, Director, Emerald Group Publishing

Lisa Spiro, Executive Director of Digital Scholarship Services, Rice University

Micah Vandegrift, Digital Scholarship Coordinator, Florida State University 


\section{Notes:}

${ }^{1}$ Notes from our discussions are available at http://bit.ly/OSIRevolutionSolution; the presentation we gave at the meeting is at: http://journals.gmu.edu/osi/article/view/1380.

2 The Max Planck white paper established that the research libraries of Germany, France, and U.K. spend more than enough on subscriptions to cover open access publications from these productive countries: Schimmer, R., Geschuhn, K. K., \& Vogler, A. (2015). Disrupting the subscription journals' business model for the necessary large-scale transformation to open access. http://dx.doi.org/10.17617/1.3. 